\title{
Videostroboscopic and morphological aspects of voice disturbances in patients with larynx atrophy and coexisting hypopharynx cancer
}

\author{
Bożena Kosztyła-Hojna ${ }^{1}$, Anna Andrzejewska ${ }^{2}$, Diana Moskal ${ }^{\mathbf{1}}$, \\ Joanna Kasperuk ${ }^{1}$, Dawid Falkowski ${ }^{1}$, Marek Rogowski ${ }^{3}$ \\ ${ }^{1}$ Department of Clinical Phonoaudiology and Logopedics, Medical University of Bialystok, Poland \\ ${ }^{2}$ Department of Medical Pathomorphology, Medical University of Bialystok, Poland \\ ${ }^{3}$ Department of Otolaryngology, Medical University of Bialystok, Poland
}

\begin{abstract}
Vocal folds play a crucial role in voice production. The physiological vibrations of vocal folds depend on the unchanged multilayered structure of the vocal folds mucosa. Morphological changes of mucosa are the cause of voice quality disorders - dysphonia. The aim of this study was to determine the morphological base of dysphonia in patients with vocal folds atrophy. A group of 24 patients with larynx atrophy confirmed by endoscopic (VLS) and stroboscopic (VLSS) examination of the larynx was included in the study. The morphological assessment of the larynx mucosa was carried out with the use of the transmission electron microscopy (TEM). Ultramorphological examinations revealed changes in the epithelium, basal membrane and lamina propria of the vocal folds mucosa. An increased number of collagenous fibers, fibroblasts with signs of vacuolar degeneration inflammatory cells and a decreased number of blood vessels and pericytes were observed. Morphological changes found in the epithelium, basal membrane and lamina propria of the vocal folds mucosa were the cause of disorders of vocal folds vibrations registered in the stroboscopic examination of the larynx (VLSS). (Folia Histochemica et Cytobiologica 2011; Vol. 49, No. 4, pp. 659-663)
\end{abstract}

Key words: larynx, vocal fold mucosa, larynx atrophy, lamina propria, videolaryngostroboscopy (VLSS), transmission electron microscopy (TEM)

\section{Introduction}

Voice quality disorders are known as dysphonia $[1,2]$. Voice pathology is caused by functional and organic changes observed in the larynx [1-3]. Vocal folds play a significant role in voice production. Pathological changes in the vocal folds mucosa result in disturbances of their normal vibration and cause intensified hoarseness. The high quality of a voice is conditioned by the multilayered and physiological struc-

Correspondence address: B. Kosztyła-Hojna,

Department of Clinical Phonoaudiology and Logopedics,

Medical University of Bialystok,

J. Kilinskiego Str. 1, 15-089 Bialystok, Poland;

tel.: (+ 48 85) 74856 52, fax: (+ 48 85) 74856 51;

e-mail: fono@umwb.edu.pl ture of the vocal folds [4, 5]. All morphological changes in lamina propria of the vocal folds mucosa are the cause of organic dysphonia.

The aim of this study was to determine the morphological changes of vocal folds of the larynx in patients with vocal folds atrophy causing dysphonia.

\section{Material and methods}

A group of 24 patients with vocal folds atrophy and coexisting hypopharynx cancer (carcinoma recessus piriformis) was included in the study. The neoplastic process referred to hypopharynx and was an indication to total laryngectomy. The analyzed group consisted of 19 (79\%) male and $5(21 \%)$ female patients aged 49 to 57 years (average 54) (Table 1).

In laryngological diagnosis, indirect laryngoscopy was used in order to establish the localization of tumor in the 
Table 1. Clinical characteristics of analyzed groups on the basis of stroboscopic and morphological material

\begin{tabular}{|c|c|c|c|c|}
\hline \multirow[t]{4}{*}{ Parameters } & \multicolumn{4}{|c|}{ Group } \\
\hline & \multicolumn{2}{|c|}{ I (larynx atrophy) } & \multicolumn{2}{|c|}{ II (control) } \\
\hline & \multicolumn{2}{|c|}{ Sex } & \multicolumn{2}{|c|}{ Sex } \\
\hline & Females & Males & Females & Males \\
\hline Material after surgery & 5 & 19 & 2 & 6 \\
\hline Autopsy material & 0 & 0 & 2 & 0 \\
\hline Number of patients (\%) & $5(21 \%)$ & $19(79 \%)$ & $4(40 \%)$ & $6(60 \%)$ \\
\hline Age span & $52-56$ & $49-57$ & $35-58$ & $49-58$ \\
\hline Average & 53 & 55 & 51 & 56 \\
\hline Total & \multicolumn{2}{|c|}{24} & \multicolumn{2}{|c|}{10} \\
\hline
\end{tabular}

hypopharynx. The assessment of larynx and the vocal folds motion was carried out by endoscopic and stroboscopic examination with the use of videolaryngoscopy by Wolf, with 90-degree optics from the same manufacturer (VLS, Videolaryngoscopy and VLSS, Videolaryngostroboscopy). The examination of the vocal folds vibration was conducted taking into consideration the vibration amplitude, mucosal wave and the phase of phonatory glottic closure. The morphological assessment was carried out using transmission electron microscopy (TEM). The material for the morphological examination was obtained from the larynx by cutting the intermembranous part of the vocal folds. The specimens of larynx were fixed in $2.5 \%$ glutaric aldehyde in kakodylate buffer, $\mathrm{pH}=7.4$ for $4 \mathrm{hrs}$ and postfixed in $1 \%$ $\mathrm{OsO}_{4}$ for 2 hrs and mounted in Epon 812. Half-thin specimens were stained with methylene blue and preliminarily evaluated using light microscopy. Ultrathin specimens were cut using a Reichert Ultracuts ultramicrotome, contrasted with lead citrate and uranyl acetate, and subsequently analyzed using a transmission electron microscope Opton 900 $-\mathrm{PC}$.

Group I included patients with vocal folds atrophy and coexisting hypopharynx cancer.

Group II - the control group - consisted of ten patients, $4(40 \%)$ female and $6(60 \%)$ male, aged 35-58 (average 54). In the material for the morphological examination, $20 \%$ of the specimens were obtained from autopsy and $80 \%$ were collected after surgical treatment. After surgery in patients with coexisting hypopharynx cancer which was an indication to total laryngectomy, no pathology of the vocal folds was observed.

\section{Results}

In Group I, the patients with vocal folds atrophy, indirect laryngoscopy revealed thin vocal folds with decreased stiffness and incomplete phonatory glottic closure.
In Group II, the vocal folds were smooth, regular, and capable of movement during respiration and phonation, with physiological phonatory closure of the glottis.

In Group I, patients in stroboscopic and endoscopic examination showed a decrease of vibration amplitude, limited mucosal wave, and a lack of phonatory closure of the glottis (Figure 1).

In Group II of patients, vocal folds vibrations were regular and appeared simultaneously, with average amplitude with the presence of normal mucosal wave and physiological phonatory closure of the glottis. (Figure 2).

In Group I, a large number of collagenous fibers (C), inflammatory cells and fibroblasts (F) with signs of vacuolar degeneration in the stroma were observed (Figures 3-7).

A small number of blood vessels exhibited multiplications of the basal membranes (BM) (Figure 4). The folds of the epithelial basal membrane were smaller in comparison with those in the control group - Group II.

In the material of Group II, we observed a multilayered flat epithelium with folds in the basal membrane, a large number of blood vessels, numerous pericytes $(\mathrm{P})$ and a small number of collagenous fibers $(\mathrm{C})$ (Figures 8-10).

\section{Discussion}

The vibration of vocal folds during phonation is conditioned by the physiological multilayered structure of the vocal folds mucosa. The lamina propria consists of a superficial layer, situated directly under the epithelial basal membrane, an intermediate layer and a deep layer $[4,5]$. The superficial layer includes a relatively small number of dispersed collagenous and elastic fibers and creates the free space prone to ede- 


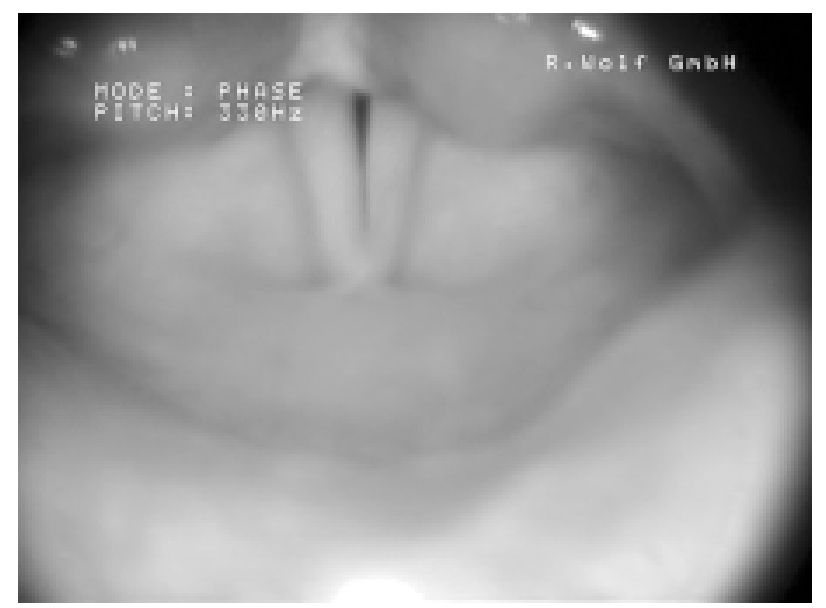

Figure 1. Atrophy of the vocal folds mucosa (VLS) — Group I

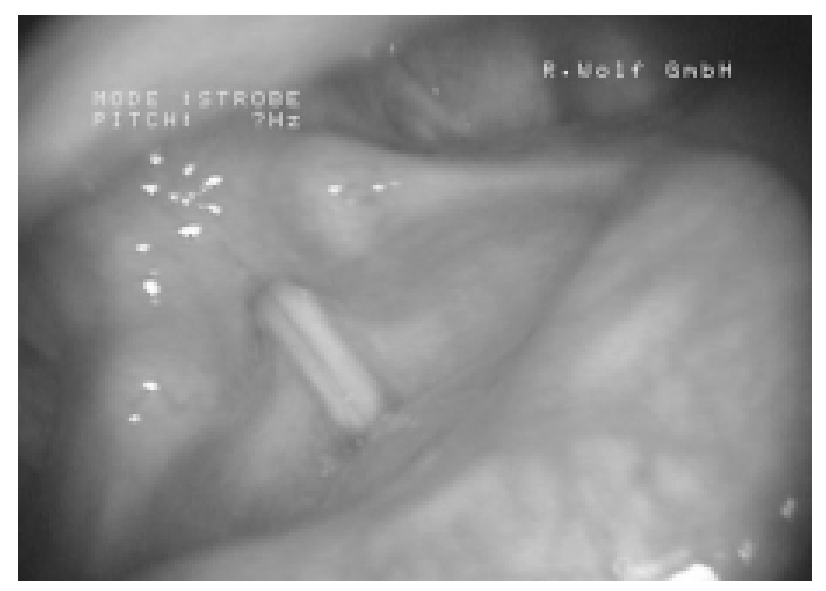

Figure 2. Physiological endoscopic findings of the vocal folds mucosa (VLS) - Group II

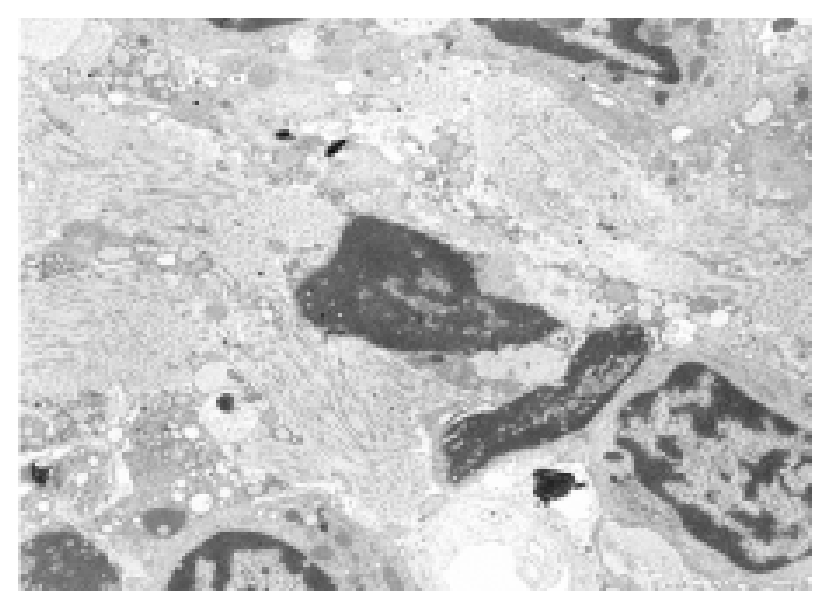

Figure 3. A large number of collagenous fibers (C) and inflammatory cells in the stroma - Group I (TEM, magnification $\times 3,000$ )

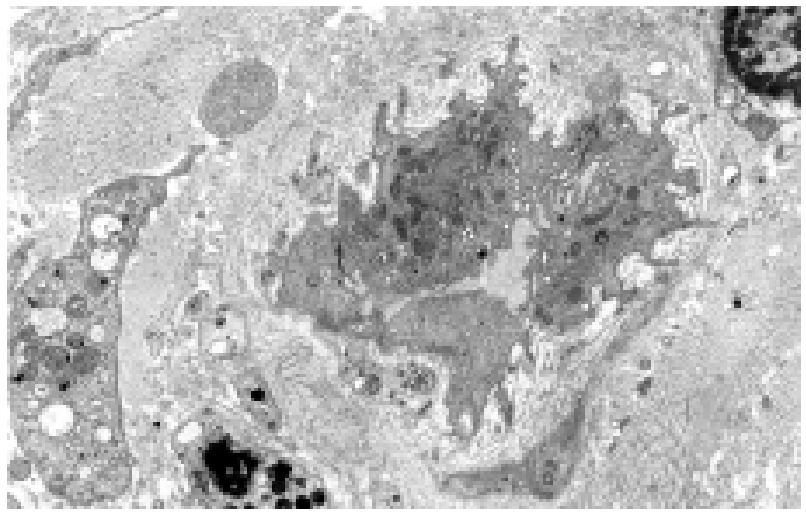

Figure 4. Multiplication of the basal membrane (BM) of the blood vessel. Signs of vacuolar degeneration of fibroblasts - Group I (TEM, magnification $\times 3,000$ )

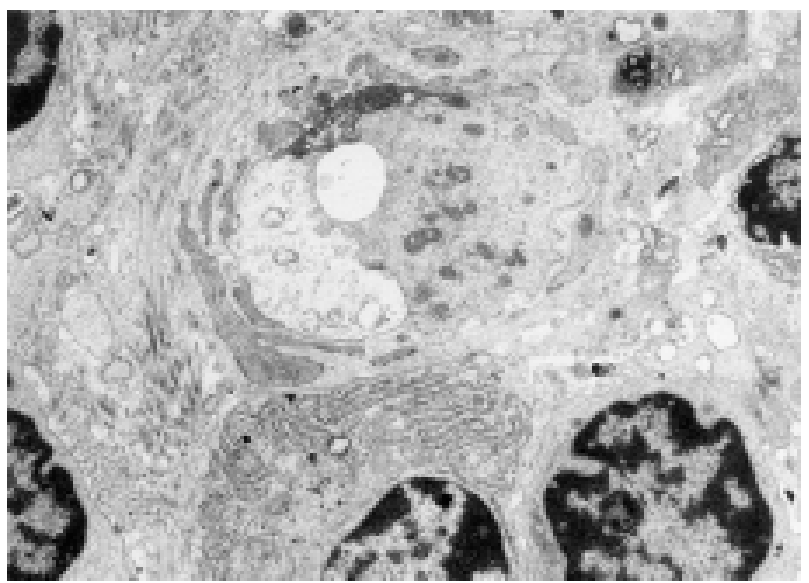

Figure 5. Numerous inflammatory cells in the stroma — Group I (TEM, magnification $\times 3,000$ )

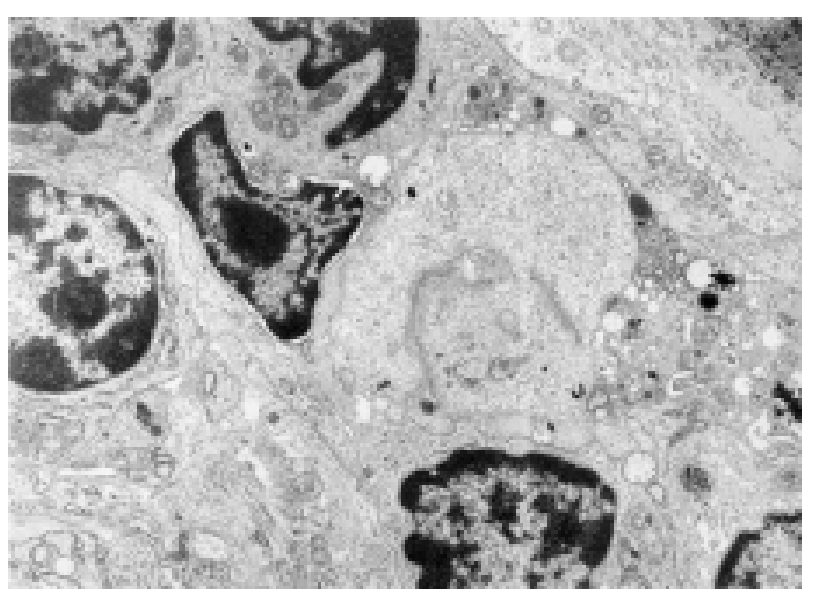

Figure 6. Lymphocytic infiltrations in the stroma — Group I (TEM, magnification $\times 3,000)$ 


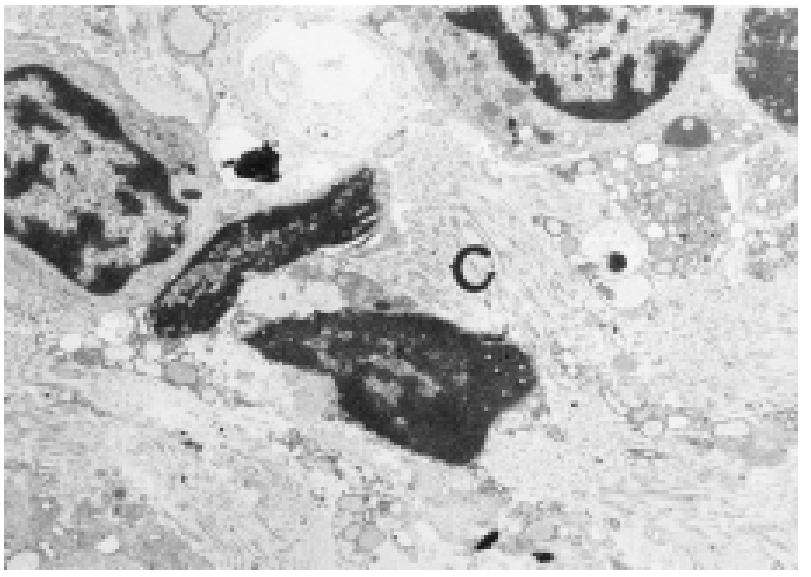

Figure 7. A large number of collagenous fibers (C) and inflammatory cells in the stroma - Group I (TEM, magnification $\times 3,000$ )

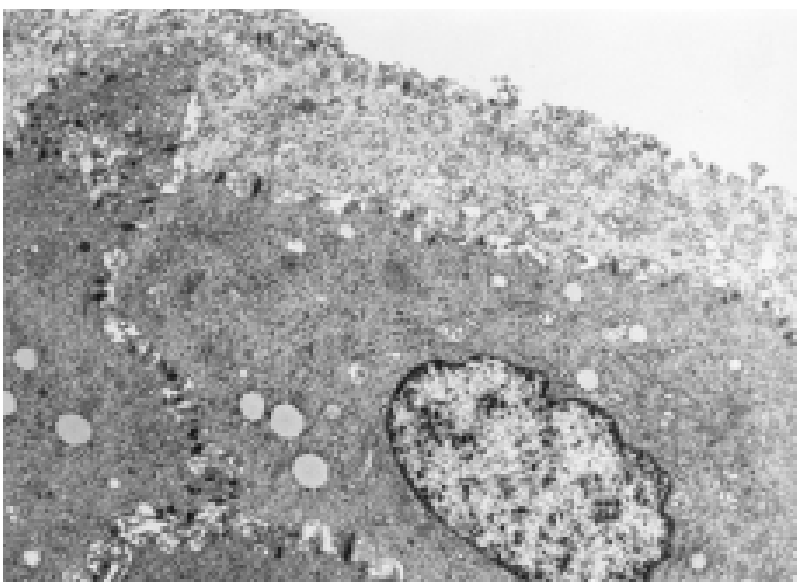

Figure 8. Epithelial cells without significant ultrastructural changes - Group II (TEM, magnification $\times 3,000$ )

ma. The deep layer of lamina propria contains a rich, thick and solid net of collagenous and elastic fibers constituting the conus elasticus. The intermediate layer includes free situated collegenous and elastic fibers.

To determine the physical factors causing dysphonia connected with vocal folds atrophy, morphological examinations of vocal folds mucosa should be performed. Hirano et al. [5] described the thinness of vocal folds of the larynx in male patients after the age of 70. According to Gray and Morrison [6, 7] vocal folds edema is more common in elderly female patients, whereas in men the atrophic form appears more frequently. Edematous changes refer mostly to the superficial layer of the lamina propria. Atrophy of the vocal folds mucosa is caused by an increase in the number of collagenous fibers in the intermediate and deep layers and a decreased number of elastic fibers [8-11]. Investigations performed by Sato [11-13] into the pres-

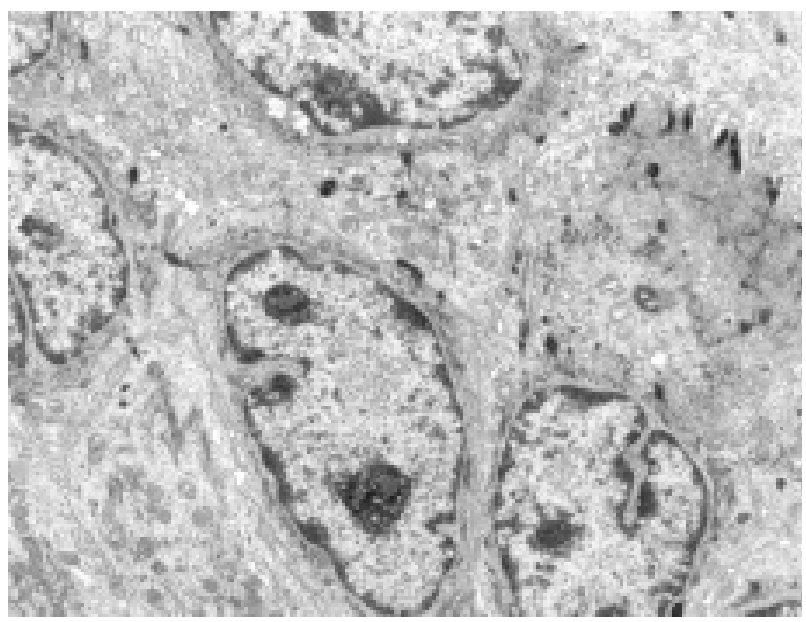

Figure 9. Epithelial cells - Group II (TEM, magnification $\times 3,000)$

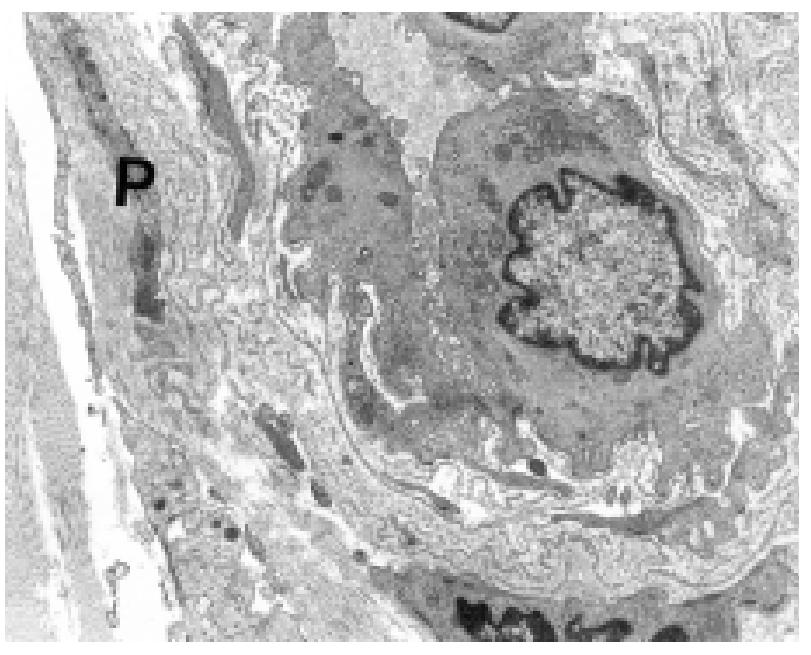

Figure 10. The vocal folds mucosa - Group II. Numerous branches of pericytes $(\mathrm{P})$. A fragment of the blood vessel with the multiplication of basal membrane (BM)

$($ TEM, magnification $\times 4,400$ )

ence of pericytes around vessels in the vocal folds mucosa indicated a large number of pericytes, of which branches make connections with endothelial vessels cells. They play a significant role in supporting vascular walls in vibrating vocal folds tissues [14].

In the analyzed material, a large number of pericytes was observed in patients of the control group without signs of atrophy of vocal folds mucosa, which was confirmed by endoscopic and stroboscopic assessment of the larynx (Figure 2). In this group of patients, the physiological mucosal wave, normal vibration amplitude and the physiological phonatory closure of the glottis were observed [1-3, 15-18]. Moreover, in epithelial cells, no significant ultrastructural changes were registered. The basal membrane showed 
folds under the epithelium. In the stroma, a large number of blood vessels and pericytes but few collagenous fibers were found. The presented ultramorphological findings suggest a lack of pathological changes $[4,10,14,19]$.

In the group of patients with atrophy of the vocal folds mucosa, the indirect laryngoscopy, endoscopic (VLS) and stroboscopic (VLSS) examinations revealed the thinness of vocal folds and incomplete phonatory closure of the glottis. The parameters of stroboscopic assessment pointed to a decrease of vibration amplitude and limited mucosal wave with incomplete phonatory glottic closure.

The presented changes confirm the clinical diagnosis of atrophy of the vocal folds mucosa and are in accordance with the studies by Kosztyła-Hojna, Vasilenko, Yanagisawa, Zalesska-Kręcicka, Rubin et al. [3, 16$-18,20]$. In the morphological material in this group of patients, a large number of inflammatory cells in the stroma, signs of vacuolar degeneration of fibroblasts, multiplications of basal membranes of blood vessels and numerous collagenous fibers were described. The folds of the epithelial basal membrane were less pronounced in comparison with the group of patients without registered pathological changes in the larynx.

The presence of morphological changes of the vocal folds mucosa in the epithelium, basal membrane and lamina propria of the vocal folds mucosa causes disturbances of the physiological vibration of vocal folds. Irregular oscillations of vocal folds have a negative impact on voice quality, causing clinical symptoms of hoarseness and roughness of voice in patients with vocal folds atrophy.

\section{Conclusions}

1. Stroboscopic parameters in the assessment of the larynx allow an objective diagnosis of atrophy of the vocal folds mucosa.

2. Ultramorphological changes in the epithelial basal membrane and lamina propria of the larynx mucosa are the cause of atrophy.

3. An increased number of collagenous fibers, fibroblasts with signs of vacuolar degeneration inflammatory cells and a decreased number of blood vessels and pericytes in lamina propria of mucosa, condition the clinical form of larynx atrophy.

\section{References}

1. Pruszewicz A. Larynx physiology. In: Pruszewicz A (ed). Clinical Phoniatrics. Warsaw: PZWL; 1992:36-47.

2. Pruszewicz A. Methodology of phonatory organ examination. In: Pruszewicz A (ed). Clinical Phoniatrics. Warsaw: PZWL; 1992:110-128.

3. Kosztyła-Hojna B, Rogowski M. Usefulness of videolaryngostroboscopy in the diagnosis of laryngeal pathology. Pol Merk Lek. 2003;14.83:413-416.

4. Hirano M. Morphological structure of the vocal folds as a vibrator and its variations. Folia Phoniatr. 1974;26:89-94.

5. Hirano M, Koike Y, Hirose K, Kasuya T. Observation of mucous membrane of human vocal cords under electron microscopy. J Otolaryngol Jpn. 1974;77:650-660.

6. Gray SD, Hirano M, Sato K. Molecular and cellular structure of vocal fold tissue. San Diego: Singular Publishing Group; 1993.

7. Morrison MD, Gore-Hickman P. Voice disorders in the elderly. J Otolaryngol. 1986;15:231-234.

8. Biondi S, Zappala M. Surface of the mucosa seen through the scanning electron microscope. Folia Phoniatr. 1974;26:241-248.

9. Bodlej HD, Wood RL. Ultrastructural studies on elastic fibers using enzymatic digestion of thin section. Anat Rec. 1972;16:71-88.

10. Frenzel H, Klainsasser O. Ultrastructural study on small blood vessels of human vocal cords. Arch Otorhinolaryngol. 1982;236:147-160.

11. Sato K, Hirono M. Age-related changes of elastic fibers in the superficial layer of the lamina propria of vocal folds. Ann Otol Rhinol Laryngol. 1997;106:44-48.

12. Sato K, Sakaguchi S, Kurita S, Hirano M. A morphological study of aged larynges. Larynx Jpn. 1992;4:84-94.

13. Sato K, Hirono M. Fine three-dimensional structure of pericytes in the vocal fold mucosa. Ann Otol Rhinol Laryngol. 1997;106:490-494.

14. Shimada T, Kitamura H, Nakamura M. Three-dimensional architecture of pericytes with special references to their topographical relationship to microvascular beds. Arch Histol Cytol. 1992;55:77-85.

15. Honjo I, Isshiki N. Laryngoscopic and voice characteristics of aged persons. Arch Otolaryngol. 1980;106:149-150.

16. Vasilenko I, Iwanchenko GF. Videolaryngoscopy and videolaryngostroboscopy in phoniatrics. Vestn Otorinolaryngol. 1991;3:38-41.

17. Yanagisawa E, Yanagisawa K. Stroboscopic videolaryngoscopy: a comparision of fiberoscopic and telescopic documentation. Ann Otol Rhinol Laryngol. 1993;102:255-265

18. Zalesska-Kręcicka M, Kręcicki T, Jeleń M. Atlas of laryngeal diseases. Wroclaw: Volumed; 1995.

19. Hirano M, Sato K, Nakashima T. Fibroblasts in human vocal fold mucosa. Acta Otolaryngol (Stockh.). 1999;119:271-276.

20. Rubin JS, Sataloff RT, Korovin GS. Diagnosis and treatment of voice disorders. Third ed. Oxford: San Diego Plural Publishing; 2006. 